\title{
Human Cumulative Cultural Evolution as a Form of Distributed Computation
}

\author{
Paul E. Smaldino ${ }^{1}$ and Peter J. Richerson ${ }^{2}$ \\ ${ }^{1}$ Center for Advanced Modeling in the Social, Behavioral, and Health Sciences, Johns Hopkins University, \\ Baltimore, MD USA \\ e-mail: paul.smaldino@gmail.com \\ ${ }^{2}$ Department of Environmental Science and Policy, University of California, Davis \\ Davis, CA USA \\ e-mail: pjricherson@ucdavis.edu
}

\begin{abstract}
Cumulative culture is the engine that drives the remarkable power of the global human computer. It enables societies to act as extremely powerful computers by ratcheting up technological and other cultural innovations. Once culture can accumulate, the ability of a society to maintain and spread complex technologies is directly related to the size of the population and its connectivity with other populations, finessing the strict limits on individual intelligence. Larger and more connected societies can maintain more complex technologies. This also means that sudden isolation or a drop in population size can lead to a loss of technology. In this chapter we first discuss how cumulative culture increases the evolutionary fitness of a population of social learners. We then focus on complex technology as a marker of cumulative cultural evolution, and discuss how technological complexity increases when cultures are both more populous and more connected. We discuss the fragility of our modern complex societies in response to disasters that may shrink the population or isolate groups. We end with a discussion of how our cultural norms and institutions shape the problems tackled by the global human computer.
\end{abstract}

Keywords: cumulative culture, social learning, technology, innovation, cultural evolution, human computation 


\section{Introduction}

This chapter, like most if not all the other chapters in this book, was written and edited on a digital computer. That computer can perform incredible feats of numerical computation at blindingly fast speeds, store massive amounts of data, and be used as a tool for everything from writing to music production to scientific analysis to communication. The abilities of a digital computer, however, are insignificant next to the computational power of the network of human beings, their communication infrastructure, and the accumulated knowledge tapped into by those individuals responsible for building it. No single human being knows how to build a modern computer from scratch. Indeed, no one knows how to build a computer mouse, or a lead pencil, or many of the complex tools we rely on for modern living from scratch (Read 1958; Ridley 2010). For that matter, hardly any of us know how to make simple twostrand twisted string from local raw materials, something that practically every adult once knew how to do. A key factor that enables us as human beings to solve complex problems and achieve a level of dominance over a wide variety of environments from the desert to the arctic to the deep ocean is not simply our individual big brains, but our capacities for extreme sociality, to cooperate and learn from one another, and our ability to build on previous knowledge and to accumulate culture.

It is often said that the human brain is like a computer. It processes information, takes in input, produces output, stores and retrieves memory. Groups of people, then, are like supercomputers. They can process in parallel, allocate resources, and divide tasks to produce faster and better solutions to problems than lone individuals can manage, and that are more than the sum of the individuals' abilities were they working separately 
(Smaldino, in press). For example, Woolley and colleagues presented small groups with a number of tasks requiring different types of collaborations to solve them (Woolley et al. 2010; see also Woolley and Hashmi, this volume). Their results showed, firstly, that groups’ performance between tasks were correlated, pointing to an emergent "collective intelligence” for each group configuration, and secondly, that a group’s performance was uncorrelated with the intelligence of its individual members, but rather stemmed from their ability to communicate in a understanding and democratic fashion.

If groups of humans are like a supercomputer, then what of human cultures, which store and process the cumulative innovations and collaborations of generations of individuals? Cumulative culture allows human societies to act as super-duper computers. Humans are unique in the animal kingdom for our tremendous capacity to learn from one another. Our relatively fast and accurate imitation and willingness to teach others allows us to acquire complex skills without having to reinvent them for ourselves. Individuals sometimes improve upon the skills they have acquired and these improvements can be passed on to those who learn from the inventor. Furthermore, we are smart shoppers in the marketplace of ideas. We selectively adopt innovations from others that work better or whose use is correlated with success. Human social learning cumulatively ratchets up technology and innovation, providing groups with progressively better solutions to the problems they encounter (Tennie et al. 2009; Boyd et al. 2011). By such means Stone Age bowyers produced bows that modern engineers find to be approximately optimal designs (Alleley et al. 1992). From this perspective, the human-based genetic algorithm (Kosorukoff 2001; Grier, this volume), a computational technique in which human users are involved in both judging the fitness of problem solutions as well as suggesting novel 
solutions, is simply an application of evolutionary processes that have been driving human innovation since the ancient hominins began to make multi-part tools.

In this chapter, we will first discuss how social learning can increase the fitness of a population by allowing cultural innovations to accumulate. We will then discuss the importance of population size and social connectivity on maintaining those innovations, with a focus on the fragility of human computational systems to sudden isolation or population loss. We end with a consideration of the implications of our discussion on the design of human computation systems in the future.

\section{Roger's Paradox: Why Social Learning Is Not Enough}

In a complex world in which decisions must often be made quickly and in which skills may be difficult to acquire, individual trial-and-error learning can be overly costly in terms of time, cognitive capacity, and the potential consequences of a poor decision. A hunter-gatherer learning on his own may spend months trying to construct a hunting apparatus or, much worse, misread animal tracks and be eaten by a predator. Social learning - which includes knowledge or behaviors acquired via teaching, imitation, or social influence (e.g., you learn to play video games because your friends all hang out and play Xbox) - helps individuals to gain important skills without the time costs and risks associated with individual, trial-and-error learning. It may therefore seem obvious that the adoption of a strategy of social learning should benefit a population - increasing its fitness, in the language of evolutionary biology. Yet, it turns out that if the only benefit of social learning is to avoid the costs of trial-and-error, then social learning strategies will be indeed adopted but will not increase the fitness of the population. 
This apparent paradox was demonstrated by Rogers (1988) via a simple mathematical model. Suppose a hypothetical world in which there are two possible behaviors, either of which individuals can adopt to help them survive and reproduce. Suppose also that the environment changes periodically between two states, and that in each of these states a different behavior yields a fitness advantage over the other behavior. Finally, suppose that individuals in this world fall into two categories of learner, and that learning strategies are passed on from parents to their children. Individual learners always learn the optimal behavior for the environment, but at a heavy cost. Social learners choose an individual from the previous generation at random and adopt that individual's behavior. Because they simply copy the behavior of another individual, social learners avoid the cost of individual learning.

When social learners are rare, they will have higher fitness than individual learners, and their prevalence in the population will therefore increase. However, as social learners become more common, a social learner becomes increasingly likely to learn her behavior from a fellow social learner. The state of the environment is not stable, so social learners risk learning an out-of-date behavior, and transmitting that incorrect behavior to social learners in subsequent generations. Thus, as the prevalence of social learners increases, their fitness begins to fall, until it again reaches the fitness of individual learners (Fig. 1). In other words, we should expect social learning strategies to evolve in a social species, but the introduction of social learning behavior does not, in and of itself, increase the average fitness of populations of that species. Boyd and Richerson (1995) studied variations on Rogers' model, including ones in which social learners could identify and preferentially learn from individual learners and ones in which 
there were more than two behaviors. They showed that Rogers' results were robust:

social learning on its own does not increase the mean fitness of a population.

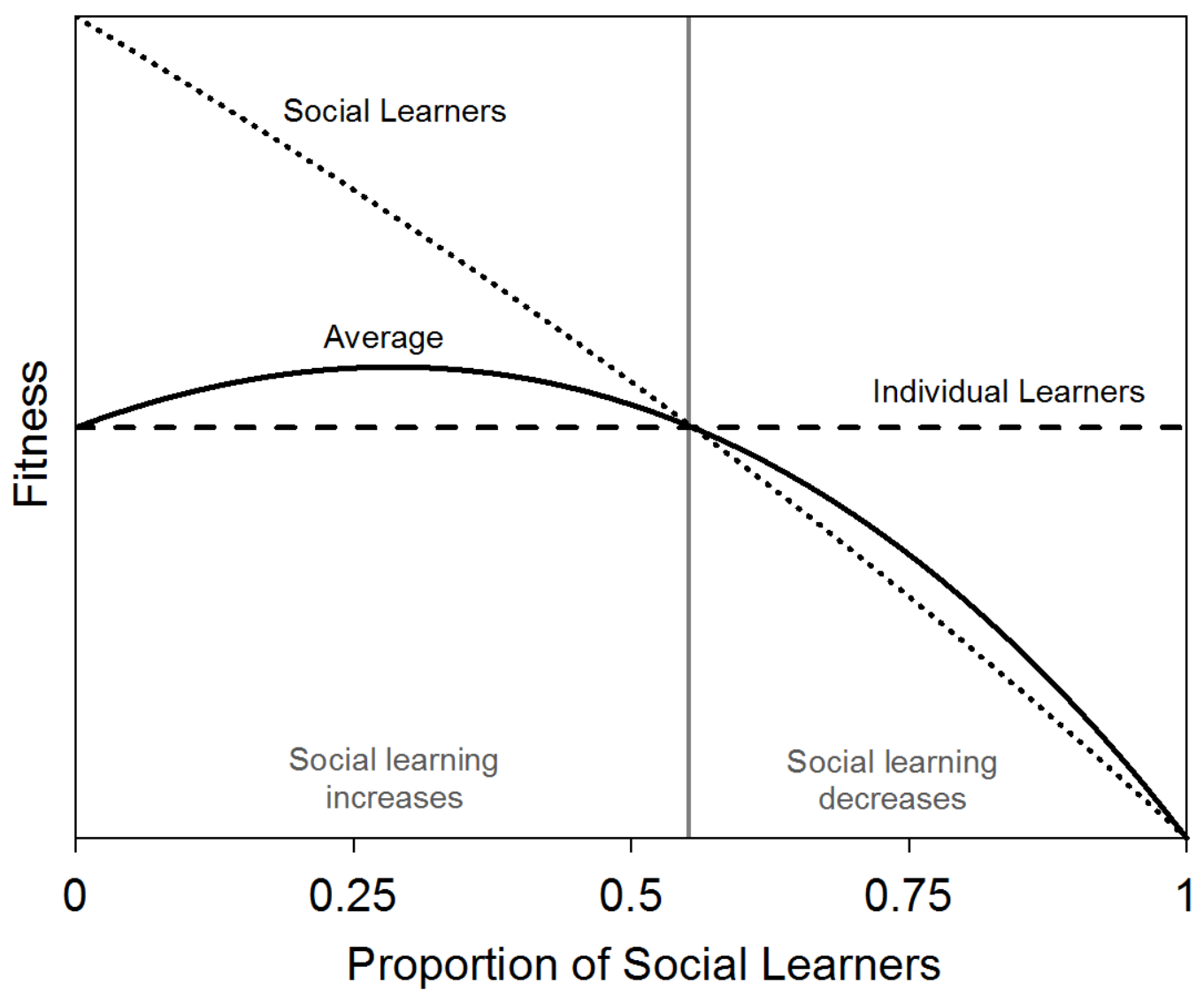

Fig. 1. Rogers' model. Social learners have higher fitness than individual learners when rare, and their invasion briefly increases the average fitness of the population. However, as the proportion of social learners in the population increases, the population stabilizes at a mixed equilibrium (vertical line) in which the average fitness is identical to that of a population of individual learners. Adapted from Rogers (1988).

\section{Cumulative Culture}

One way of getting around Roger's paradox is if social learning can improve the efficiency of individual learning. For example, suppose individuals sample multiple behaviors, and choose one if it is obviously better than the others. If there is an insufficiently clear signal, and one behavior is not obviously superior, then the individual 
chooses a random behavior through imitation. This strategy of "conditional social learning” has been shown to increase the mean fitness of the population relative to a population of individual learners under a variety of conditions (Boyd and Richerson 1995; Enquist et al. 2007; Ehn and Laland 2012). In this and related scenarios, behaviors can always be hypothetically learned by individuals through trial and error. Social learning can be adaptive if it hastens the spread of beneficial behaviors. Indeed, this seems to be the primary benefit of social learning in non-human apes (Tennie et al. 2009). Nonetheless, human groups have utilized social learning to make extremely large adaptive gains, much larger than would be facilitated solely through the increased spread of beneficial behaviors. The key to human success is the spread of incremental innovations based on existing behaviors - often quite complex behaviors - which naïve individuals would be unable to learn on their own. Human social learning based on teaching and imitation is so efficient that a Stone Age bowyer could acquire an advanced technology like a bow that already incorporated the hard-won innovations of dozens if not hundreds of his ancestors before he contemplated innovations that might improve it still further.

Humans are the most successful vertebrate species on the planet. We have managed to conquer a vast range of environments from the desert to the tropics to the Arctic. We have modified our environments to facilitate our survival and our expansion. At the dawn of the agricultural revolution 10 thousand years ago, the entire human population across the globe was approximately 5 million (Keinan and Clark 2012). Today, over 37 million people live in the Tokyo metropolitan area alone. We have achieved this success through the process of cumulative culture. By this, we mean that 
learned information and behaviors are reliably transmitted and improved upon, such that those improvements can in turn be transmitted through learning.

Cumulative culture allows individuals to build on previous adaptations. To exploit this, humans have evolved psychological mechanisms, heuristics, and biases that facilitate the acquisition of useful knowledge and behaviors (Henrich and McElreath 2003; Tomasello et al. 2005; Herrmann et al. 2007; Hill et al. 2009). This includes learning from individuals who have demonstrated success either directly or by proxy, indicated by traits like status or prestige (Henrich and Gil-White 2001). Symbolic communication additionally allows for transmission of behaviors and practices without direct observation. Stories, myths, and moral doctrines play an important role in the cultural transmission of norms of social behavior as well as useful information about survival (Chudek and Henrich 2011). Moreover, the spread of organizational norms through narratives, religions, and other social institutions have enabled the creation and transmission of emergent group-level traits that rely on social organization and division of labor (Henrich and Boyd 2008; Smaldino, in press). The capacities of human populations for the storage and transmission of collective information constitute a feat of evolutionary computing unmatched in the natural world.

\subsection{Why culture is common but cultural evolution is rare}

Since cumulative culture has made our species so outstandingly successful, why didn’t this capacity evolve long ago and lead to many highly cultural species? After all, most "killer" adaptations like internal skeletons, camera-type eyes, and efficient flight have been around for hundreds of millions of years and characterize many lineages. At 
least two answers are possible. First, cumulative culture would be very difficult to get started if the capacity for it is costly, as our large brains suggest it is (Aiello and Wheeler 1995). The problem is that it takes many large brains operating over many generations to evolve complex cultural adaptations. The first individuals to pay the cost for a capacity for cumulative culture would find no useful complex traits to imitate and hence would get no fitness payoff to cover the overhead of the capacity (Boyd and Richerson 1996). Second, the kind of environment that makes complex cumulative culture useful may be of very recent vintage. Theoretical models suggest that cumulative culture is most useful in moderately variable environments, especially environments that vary on time scales too short for genes to track. Cultural evolution is rapid compared to genetic evolution and can thus generate adaptations to more ephemeral environmental changes than can genes (Perreault 2012). We have known for decades that the earth's climates became much more variable during the Plio-Pleistocene - from 5.3 million years ago to about 11.5 thousand years ago - than they were during the preceding 60 million years (Zachos et al. 2001). Until recently, however, the best data described climate variation on time scales too long to favor costly culture. As better paleoclimate data has come available, we have begun to resolve climate variation at the millennial and submillennial scale that in theory should favor a capacity for cumulative culture. This variation appears to have been increasing over the last few glacial cycles (Loulergue 2008), in rough parallel to human brain size increase and the increasing sophistication of stone tools. In other words, paleoclimate data for the last several hundred thousand years shows a steady increase in environmental variability that appears to be tracked by the emergence and spread of 
cumulative culture.

\section{Innovation, Environment, and Population Size}

The ability to socially learn and accumulate culture provides opportunities for the expansion of humans’ ability to problem-solve. However, the ability to learn and adapt does not necessarily lead to the runaway growth of cultural innovation. As a prime example, anatomically modern humans first appeared in Africa between 160 and 200 kya (kya = thousand years ago; McBrearty et al. 2000; White et al. 2003; McDougall et al. 2005), and had spread to most of the habitable parts of the globe between 90 and 40 kya (Ambrose 1998; Ray et al. 2005). Yet the appearance of agriculture and the comparatively rapid growth of culture, technology, and population size only occurred around 11 kya. Why is this the case? It seems unlikely that it took somewhere between 30 and 150 thousand years for humans to happen upon the idea of domesticating crops and adopting stationary (as opposed to nomadic) lifestyles. Richerson et al. (2001) have argued that the drier, highly variable, low $\mathrm{CO}_{2}$ world of the last glacial period would have been unfavorable for the evolution of agriculture until about 11,000 years ago, which is in fact when agriculture began to develop. It is true that anatomically modern humans were present in Africa during the last interglacial period without developing agriculture, and our understanding of events in Africa leading up to anatomical moderns spreading out of Africa around 50 kya is still rudimentary (Richerson et al. 2009). Nevertheless, the case is strong that it was the right mix of biological and cultural preparedness and worldwide climatic factors that triggered the widespread adoption of agriculture, sowing the seeds of modern civilization. 
Even in the relatively stable global climate of the last 11,000 years, cultural innovations have required the right social and environmental circumstances to thrive and evolve. One of the best markers of cumulative cultural evolution is the presence of complex technology. Before most of the world was connected by webs of communication and commerce, tremendous variability could be found in the complexity of each culture's toolkits. What factors determine the limits of a cultural population’s technological complexity?

\subsection{Population size and connectedness predict technological complexity}

Cumulative culture allows for complex technologies to be maintained and transmitted across generations. As a result, humans have developed technologies that have allowed them to survive and flourish in wide ranges of environments. Spears and kayaks are useful for fishing in marine environments, but would be quite difficult for naïve individuals to make and exploit on their own. Inuit populations living in Arctic climates learned to make warm skin clothing, build sleds, and breed and train dogs for sled travel. All of these cultural adaptations were essential for their long-term survival in the icy climate of the frozen North. The creation of all of these technologies depends on the ability to transmit and maintain complex and cumulative information and behaviors. However, learning complex behaviors and technologies is not easy. For example, not everyone is a great model for learning. In a generation, there may be only one or two great kayak builders. Moreover, not everyone has the ability or the proclivity to learn complex skills. From among many students, a gifted teacher may have only a few gifted 
pupils who can go on to teach others to the same high standard. How might these factors influence the maintenance and transmission of complex cultural adaptations?

Using a mathematical model, Henrich (2004) showed that if (1) there is individual variation in learning ability, and (2) students are usually less skilled than their teachers, then more complex skills will require larger population sizes in order to be maintained. Powell et al. (2009) then extended Henrich’s model and showed that it is not necessarily the absolute size of the population that matters, but rather the number of effective teachers available. Thus, contact with other groups can compensate for a given group’s small population size. On the other hand, this theory implies that smaller and more isolated groups should have less complex technology than larger and more connected groups. Kline and Boyd (2010) analyzed fishing and marine foraging toolkits from 10 small-scale societies in Oceania and found that, as the models predicted, population size was the best predictor of toolkit complexity, and also that higher rates of contact with other groups were associated with greater toolkit complexity, especially in relatively small groups.

\subsection{When disaster strikes}

If larger and more connected populations are associated with increased technological complexity, what happens if there is a catastrophic event that suddenly shrinks the population or isolates a group from outside contact? Several documented cases suggest that this can lead to a loss of previously held technologies (Boyd et al. 2011). A well-known example is the case of the Tasmanians (Diamond 1978; Henrich 2004; Davidson and Roberts 2009). Isolated from mainland Australia after the seas began 
to rise at the end of the last glacial period, humans on Tasmania were stranded for about 8,000 years on an island that could not sustain more than a few thousand people, and remained isolated from the rest of the world until their first contact with Europeans in the late eighteenth century (Pardoe et al. 1991). Over the next several thousands of years following their isolation, the Tasmanians lost a number of previously held technologies, such as bone tools and fishing hooks and the ability to make cold-weather clothing, which were nevertheless maintained in Aboriginal communities on the Australian mainland. Moreover, the archaeological record on Tasmania points to a gradual loss of technology following their isolation. For example, the record indicates that between 8,000 and 5,000 years ago the Tasmanian diet was heavily dependent on fish. However, the presence of fish in their diet (as seen in the archaeological record) was declining by 5,000 years ago and had completely disappeared by 3,800 years before present, even though the relative proportions of other elements in the Tasmanians' diet did not shift much. Other technologies that appeared on the mainland after the Tasmanian separation may have never arisen on the island at all. For example, boomerang use was widespread on the mainland by the time of European contact, and the remains of boomerangs dating back to over 11,000 years ago have been found in a peat bog in South Australia, but none have ever been found in Tasmania (Davidson and Roberts 2009).

\section{Technological complexity in the modern world}

The past two centuries have seen exponential growth in both the size of the human population on Earth and the complexity of our technology. Each new technological innovation has built upon previous or contemporary technologies, and 
those innovations have spread with startling speed. Consider that a person alive today born 100 years ago would have witnessed the inventions of - just to name a few - the television, the transistor radio, modern plastics, the jet airplane, the electric guitar, the microwave, the credit card, the remote control, the compact disc, personal computers, cell phones, the internet, GPS, DNA fingerprinting, Prozac, Viagra, gene sequencing, smart phones, and unmanned drone aircraft. Consider also that in that time, the world population has not only quadrupled, but has become vastly more connected. Threefourths of the world's people now have access to cell phones (World Bank 2012). Social media such as Facebook, Twitter, and Google+ allow information, ideas, and norms to spread faster and wider than ever before. The research university and other formal educational and R\&D organizations spread technical information to ever larger numbers of students and support ever larger numbers of research scientists, design engineers, manufacturing specialists and maintenance technicians.

Access to advanced technologies is extremely widespread. However, the knowledge and ability to create innovative new technologies rests in the hands of rather few individuals in each separate field. Moreover, many technologies require complicated collaborations between individuals with different skill sets and access to resources - a prerequisite fulfilled by large numbers of people with access to communication networks and substantial wealth. As our population grew and became more interconnected, our capacities to invent and sustain complex technologies increased. However, the maintenance of those technologies is not guaranteed. For example, it may be that the number of highly trained engineers necessary to sustain (much less advance) modern highly complex technology is quite large. If the global human computer were to suffer 
some kind of setback (due to a political, economic, or environmental shock), the loss of individual experts or the reduced communication between them might further exacerbate the original setback, much as a shrinkage of population size and/or contact caused the loss of complex technology on Tasmania and on remote Pacific islands. As an example from a complex society, consider also the setbacks to technology and knowledge in the former Western Roman Empire after its political collapse. Depopulation and the fragmentation of the formerly unified polity and economy resulted in declines in literacy and the hollowing out of civil engineering skills so advanced by the Romans. Old buildings and roads fell into ruin and new construction did not recover to Roman standards until the Late Medieval period.

\subsection{How fragile is the global human computer?}

Could a disaster characterized by a sudden loss of either population or connectedness really lead to a global loss of technology? Maybe not. The world is extremely well connected. This may create redundancies and plasticity that could prevent loss of technology, provided the damage was not too extensive. Consider an analogy to the human brain. Damage to the left hemisphere of the cerebral cortex, such as caused by a stroke or traumatic brain injury, can cause difficulties in the production or comprehension of language (aphasia). However, if a patient speaks more than one language, she may show differential patterns of damage and recovery between languages, making the patient more likely to regain or retain language use in at least one language (Goral et al. 2002). Increased social connections whereby similar technologies are produced via slightly different pathways may increase the robustness of complex 
technologies. We also have large information reserves in the forms of books and internet databases. Although the Romans had books, the printing press had not yet been invented, and as such dissemination of information was limited. Merlin Donald (1991) has argued that literacy and numeracy, leading to the external storage of information, is one of the great advances (of three) in the origin of the modern mind. The unaided human brain has a limited memory and a limited ability to handle quantitative calculations. Literacy and numeracy not only relieve these limitations but also increase connectedness via books and other forms of written communication. Mass literacy and inexpensive mass media following the invention of the printing press greatly multiplied the number of people who could participate in advancing and spreading technology and other innovative ideas.

Nevertheless, it is possible that technology may be lost should disaster strike. Much of our specialized knowledge is collected by institutions, and that knowledge could rapidly vanish. Skilled people can die, books can be burned, and computers can wear out. Cumulative culture creates infrastructures that facilitate the persistence and growth of technologies and innovations. The maintenance of modern medicine, for example, leans on the infrastructure of the medical school system as much as it does on the availability of information. If young would-be doctors didn’t have anywhere to train, it would be difficult for them to become as skilled as today’s highly trained doctors regardless of the persistence of medical textbooks. If the population necessary to maintain a particular technology were to suffer a loss, it is not clear that recovery would be swift.

Even if a technology is lost, knowledge of the technology's existence and its associated benefits may help to recover it. The archaeological evidence shows that for thousands of years following their separation from the mainland, the Tasmanians' diet 
involved large quantities of fish. When European's visited the island, however, the islanders were astonished at their success in pulling fish out of the ocean, but refused offers of fish to eat (Davidson and Roberts 2009). This taboo on eating fish almost certainly arose after the islanders stopped fishing, along with their loss of the technology to make bone fishing hooks. Importantly, the taboo would have also dissuaded potential innovators from re-inventing fishing equipment, or prevented such technology from spreading should it have arisen. While this taboo may have helped to concentrate efforts on the acquisition of land-based sources of food, it also potentially halted technological innovation. In contrast, some of the more recent technological innovations in the modern world were driven by science fiction writers' visions of what could be. It is possible that, in the aftermath of a technology loss, recovery could be aided by visions of what once was.

\subsection{The future of innovation}

Is it possible for human computation to improve the operation of cumulative culture, so that we may avoid catastrophic technology loss altogether? We can draw insight from how it has been improved in the past, focusing on the last ten millennia and particularly on the most recent centuries and even decades. Table 1 details some of these mechanisms. Contemporary web-based efforts to speed the rate of innovation (e.g. Spigit.com, Innocentive.com, Google Scholar) are based on further improvements along the lines outlined in Table 1. Electronic storage is so cheap that we can aspire to have the sum total of human knowledge stored in electronic media. Even today a large fraction of that total is available to those of us with access to the web and a research library with 
electronic journal subscriptions. Top universities are offering free online courses and Wikipedia has authoritative micro-courses on a host of topics. Given that much innovation involves novel combinations of ideas, the ability to rapidly access a wider variety of ideas and skills should increase the rate of innovation.

Table 1. Culturally evolved mechanisms for improving the efficacy of cumulative culture.

\begin{tabular}{|l|l|}
\hline Mechanism & Examples \\
\hline Development of off-line storage of information & $\begin{array}{l}\text { Literacy, numeracy, and cheaper media such as } \\
\text { clay tablets, paper, and electronic storage. }\end{array}$ \\
\hline $\begin{array}{l}\text { Improvements in the dissemination of } \\
\text { information }\end{array}$ & $\begin{array}{l}\text { Cheap printing, lending libraries, internet } \\
\text { search engines }\end{array}$ \\
\hline $\begin{array}{l}\text { Improvements in the capacity of individuals to } \\
\text { innovate and the number of individuals } \\
\text { prepared to learn }\end{array}$ & $\begin{array}{l}\text { Mass education, specialized scientific and } \\
\text { technical occupations, specialist textbooks and } \\
\text { journals }\end{array}$ \\
\hline $\begin{array}{l}\text { Development of institutions designed to favor } \\
\text { innovation }\end{array}$ & $\begin{array}{l}\text { Intellectual property rights, research } \\
\text { universities, lavish government support for } \\
\text { basic and applied research, "Silicon Valley } \\
\text { culture” }\end{array}$ \\
\hline
\end{tabular}

Two limitations to the power of the internet to help speed innovation remain. First, intellectual property issues must be solved. Book and journal publishers have so far prevented rapid, inexpensive access to all potentially useful information, even in the realm of academic publishing where authors expect to be paid mainly in the form of prestige rather than money. Companies that crowdsource innovation, like Spigit and Innocentive, restrict access to their services to a closed community to protect intellectual property rights. Complicating the issue, the protection of intellectual property rights likely provides a key incentive for innovators in the modern world of global interconnections. Users of information will often be strangers who will not be inclined to bestow prestige rewards on innovators, much less material rewards. Some small, simple, 
and automated per-view or per-download royalties would compensate creators of groupbeneficial innovations, who would not otherwise benefit from their work.

Second, as Polanyi (1966) argued, much knowledge is "tacit" - the fingertip feel for things that is very difficult, if not impossible, to reduce to print or pictures. For example, to educate scientists we still rely on the highly personalized, labor intensive PhD system pioneered in Germany in the early 19th Century precisely because it allows for the transfer of tacit knowledge. Similarly, firms expect to have to inculcate their organization's ethos into technical and management hires. And one might reasonably entertain the possibility that most undergraduate students prefer to attend residential universities, perhaps not entirely for the parties. Thus, the need to transmit tacit knowledge is enduring, and as such, seems to act as a fundamental limit on the rate of cumulative cultural evolution.

As we proceed into the future, what it means to improve our ability to generate and maintain cultural innovations may grow considerably less straightforward. The pace of evolution is set by the rates of dissemination and innovation. Human communities are now integrated with digital devices that are themselves excellent learners, ratcheting up our ability to innovate. With more people producing more technology with more variation than ever before, culture is evolving at increasing rates. A concern, however, is that modern cumulative culture operates much more rapidly in some areas than in others. Technology that can be adopted by an individual, for example, can often spread faster than institutions that require coordination among many individuals.

Which problems we solve with our technology will depend partly on what we perceive to be the salient problems. Culture influences how we perceive the world 
(Smaldino \& Richerson, 2012). If our problems continue to be framed in terms of the accumulation of wealth and power, then the human computational engine will surely continue to apply its problem-solving powers in that sphere. That strategy, however, will very likely lead to a catastrophe with the potential to disable the infrastructure of the global human computer that made it possible. As Nardi writes in her eloquent chapter (this volume), “There is no energy cornucopia waiting for us to tap into; we live on a specific planet, with specific resources. We are in the process of using up those resources.” A more promising direction, in terms of prolonging the existence of our impressive culture achievements, is to attempt to guide the evolutionary forces that shape cultural change toward those individual mindsets and social and legal institutions that promote forethought and sustainability .

\section{Summary}

Cumulative culture is the engine that drives the remarkable power of the global human computer. It enables societies to act as super-duper computers by ratcheting up technological and cultural innovations. Once culture can accumulate, the ability of a society to maintain and spread complex technologies is directly related to the size of the population and its connectivity with other populations. Larger and more connected societies can maintain more complex technologies. This also means that sudden isolation or a drop in population size can lead to a loss of technology. The modern world maintains highly complex technology requiring the interactions of many varied, superbly trained individuals. A catastrophic loss in terms of either life or connectivity has the potential to trigger what some may consider an equally devastating loss of technology. While our 
interconnected society may have the resources to avert such a second-order crisis, our best bet in the face of a loss of technology is to retain knowledge of its existence in our collective memory and to continue developing the kinds of human computational tools that have served us in the past. As the accelerated pace of our cultural evolution comes at the cost of increased resource use, however, we may need to focus on shifting the evolutionary forces that guide the norms and institutions that define the psychological state space of problems and solutions. It would be a shame to damage the global human computer through a product of its own doing. 


\section{References}

Aiello, L. C., \& Wheeler, P. (1995). The expensive-tissue hypothesis: The brain and the digestive system in human and primate evolution. Current Anthropology, 36, 199221.

Allely, S., Baker, T., Comstock, P., Hamm, J., Hardcastle, R., Massey, J., \& Strunk, J. (1992). The traditional bowyer's bible. Guilford, CT: Lyons Press.

Ambrose, S. H. (1998). Late Pleistocene human population bottlenecks, volcanic winter, and differentiation of modern humans. Journal of Human Evolution 34, 623-651.

Boyd, R., \& Richerson, P. J. (1995). Why does culture increase human adaptability? Ethology and Sociobiology, 16, 125-143.

Boyd, R., \& Richerson, P. J. (1996). Why culture is common but cultural evolution is rare. Proceedings of the British Academy, 88, 73-93.

Boyd, R., Richerson, P. J., \& Henrich, J. (2011). The cultural niche: Why social learning is essential for human adaptation. Proceedings of the National Academy of Sciences, 108, 10918-10925.

Chudek, M., \& Henrich, J. (2011). Culture-gene coevolution, norm-psychology and the emergence of human prosociality. Trends in Cognitive Sciences, 15, 218-226.

Davidson, I., \& Roberts, D. A. (2009). On being alone: The isolation of the Tasmanians. In M. Crotty \& D. A. Andrews (Eds.), Turning points in Australian history (pp. 18-31). Sydney, Australia: University of New South Wales Press.

Diamond, J. M. (1978). The Tasmanians: The longest isolation, the simplest technology. Nature, 273, 185-186.

Donald, M. (1991). Origins of the modern mind: Three stages in the evolution of culture 
and cognition. Cambridge, MA: Harvard University Press.

Ehn, M., \& Laland, K. (2012). Adaptive strategies for cumulative cultural learning. Journal of Theoretical Biology, 301, 103-111.

Enquist, M., Eriksson, K., \& Ghirlanda, S. (2007). Critical social learning: A solution to Rogers' paradox of non-adaptive culture. American Anthropologist, 109, 727-734.

Goral, M., Levy, E. S., \& Obler, L. K. (2002). Neurolinguistic aspects of bilingualism. International Journal of Bilingualism, 6, 411-440.

Henrich, J. (2004). Demography and cultural evolution: How adaptive cultural processes can produce maladaptive losses - the Tasmanian case. American Antiquity, 69, 197-214.

Henrich, J., \& Boyd, R. (2008). Division of labor, economic specialization and the evolution of social stratification. Current Anthropology, 49, 715-724.

Henrich, J., \& Gil-White, F. J. (2001). The evolution of prestige: Freely conferred deference as a mechanism for enhancing benefits of cultural transmission. Evolution and Human Behavior, 22, 165-196.

Henrich, J., \& McElreath, R. (2003). The evolution of cultural evolution. Evolutionary Anthropology, 12, 123-135.

Herrmann, E., Call, J., Hernández-Lloreda, M. V., Hare, B., \& Tomasello, M. (2007). Humans have evolved specialized skills of social cognition: The cultural intelligence hypothesis. Science, 317, 1360-1366.

Hill, K., Barton, M., \& Hurtado, A. M. (2009). The emergence of human uniqueness: Characters underlying behavioral modernity. Evolutionary Anthropology, 18, 187200. 
Keinan, A., \& Clark, A. G. (2012). Recent explosive human population growth has resulted in an excess of rare genetic variants. Science, 336, 740-743.

Kline, M. A., \& Boyd, R. (2010). Population size predicts technological complexity in Oceania. Proc. R. Soc. Lond. B, 277, 2559-2564.

Kosorukoff, A. (2001). Human based genetic algorithm. IEEE Transactions on Systems, Man, and Cybernetics, 5, 3464-3469.

Loulergue, L., Schilt, ., A., Spahni, R., Masson-Delmotte, V., Blunier, T., Lemieux, B., Barnola, J.-M., Raynaud, D., Stocker, T. F., \& Chappellaz, J. (2008). Orbital and millennial-scale features of atmospheric CH4 over the past 800,000 years. Nature, 453, 383-386.

McBrearty, S., \& Brooks, A. S. (2000). The revolution that wasn't: A new nterpretation of the origin of modern human behavior. Journal of Human Evolution, 39, 453563.

McDougall, I., Brown, F. H., \& Fleagle, J. G. (2005). Stratigraphic placement and age of modern humans from Kibish, Ethiopia. Nature, 433, 733-736.

Pardoe, C., Bowdler, S., Brace, C. L., Plomley, N. J. B., Turner, C. G., \& Wolpoff, M. H. (1991). Isolation and evolution in Tasmania [and comments and reply]. Current Anthropology, 32, 1-21.

Perreault, C. (2012). The pace of cultural evolution. PLOS ONE, 7, e45150.

Polanyi, M. (1966). The tacit dimension. Garden City, NY: Doubleday.

Ray, N., Currat, M., Berthier, P., \& Excoffier, L. (2005). Recovering the geographic origin of early modern humans by realistic and spatially explicit simulations. Genome Research, 15, 1161-1167. 
Read, L. (1958). I, pencil: My family tree as told to Leonard E. Read. In The Freeman; reprinted by The Library of Economics and Liberty. Retrieved from http://www.econlib.org/library/Essays/rdPncl1.html.

Richerson, P. J., Boyd, R., \& Bettinger, R. L. (2001). Was agriculture impossible during the Pleistocene but mandatory during the Holocene? A climate change hypothesis. American Antiquity, 66, 387-411.

Richerson, P. J., Boyd, R., \& Bettinger, R. L. (2009). Cultural innovations and demographic change. Human Biology, 81, 211-235.

Ridley, M. (2010). The rational optimist: How prosperity evolves. New York: HarperCollins.

Rogers, A. R. (1988). Does biology constrain culture? American Anthropologist, 90, 819831.

Powell, A., Shennan, S., \& Thomas, M. G. (2009). Late Pleistocene demography and the appearance of modern human behavior. Science, 324, 1298-1301.

Smaldino, P. E. (in press). The cultural evolution of emergent group-level traits. Behavioral and Brain Sciences.

Smaldino, P. E. \& Richerson, P. J. (2012). The origins of options. Frontiers in Neuroscience, 5, 50.

Tennie, C., Call, J., \& Tomasello, M. (2009). Ratcheting up the ratchet: On the evolution of cumulative culture. Philosophical Transactions of the Royal Society B, 364, 2405-2415. 
Tomasello, M., Carpenter, M., Call, J., Behne, T., \& Moll, H. (2005). Understanding and sharing intentions: The origins of cultural cognition. Behavioral and Brain Sciences, 28, 675-735.

White, T. D., Asfaw, B., DeGusta, D., Gilbert, H., Richards, G. D., Suwa, G., \& Howell, F. C. (2003). Pleistocene Homo sapiens from Middle Awash, Ethiopia. Nature, 423, $742-747$.

World Bank. (2012). Mobile phone access reaches three quarters of planet's population. Retrieved from http://www.worldbank.org/en/news/pressrelease/2012/07/17/mobile-phone-access-reaches-three-quarters-planetspopulation

Zachos, J. C., Shackleton, N. J., Revenaugh, J. S., Palike, H., \& Flower, B. P. (2001). Climate response to orbital forcing across the Oligocene-Miocene boundary. Science, 292, 274-278. 